Aus der I. Medizinischen Klinik der Akademie für praktische Medizin in Köln. (Direktor: Geheimrat Hochhaus.)

\section{Experimentelle und therapeutische Erfahrungen mit Diathermie.}

Von Dr. H. Dreesen, Assistenzarzt.

Diathermie oder Thermopenetration ist ein zuerst von Ze ynek, v. Bernd und v. Pre yss (1) angegebenes Verfahren, bei dem hochfrequente elektrische Ströme von erheblicher Spannung und Intensität durch den Körper geleitet und dort infolge des Widerstandes in sogenannte Joulesche Wärme umgewandelt werden. Mit dieser Methode ist es möglich, einen Körperteil nicht nur an seiner Oberfläche, sondern auch tief im Inneren zu erwärmen, ohne da ß ein elektrischer Reiz oder sonst eine unerwünschte Nebenwirkung dabei auftritt. . Die damit erzielten Resultate sowohl im Experiment als auch therapeutisch beim Menschen sind derart, daß sie zur Nachprüfung auffordern; über unsere in $1 \frac{1}{1}$ Jahren gesammelten Erfahrungen wollen wir kurz berichten. Wir benutzten den Apparat der Firma Reiniger, Gebbert \& Schall. ${ }^{1}$ )

Zunächst haben wir durch experimentelle Untersuchungen analog früheren festzustellen versucht, ob mit der Diathermie sich in der Tiefe der Organe tatsächlich eine erhebliche Wärmeentwicklung erzeugen läßt.

Nagelschmidt (2) und Laqueur (3) haben in Fleischstücken Temperatursteigerungen konstatiert. Schittenhelm (4) konnte sie an großen Hunden so hoch treiben, daß der Tod eintrat. Kling müller (5) erreichte beim Kaninchen nach Laparotomie im Abdomen Temperaturen bis 4l, $5^{0} \mathrm{C}$. Schminke (6) hat auch am. Menschen in der Hohlhand und in der Mundhöhle Wärme bis $45^{\circ} \mathrm{C}$ erhalten. Fürstenberg (7) erzielte bei Diathermie des menschlichen Magens den größten Temperaturanstieg von $0,4^{0} \mathrm{C}$ bei nur 0,3 Ampère Stromstärke; benutzte er mehr, so sank die Temperatur wieder.

Wir haben unsere Versuche in verschiedener Weise angestellt; einmal an zwei Gipsmodellen, die in der Weise hergestellt waren, da $B$ ein Gipsrohr von $4 \mathrm{~cm}$ Dicke, in dessen Lumen das Thermometer eingeschoben wurde, von einer $21 / 2 \mathrm{~cm}$ breiten Watteschicht und diese wieder mit einer $1 \mathrm{~cm}$ dicken Gipshülle umgeben wurde. An diesen Modellen - für. Wärme und Elektrizität sehr schlechte Leiter - wurde unter möglichst gleichen Bedingungen die mit Diathermie erzielte Wärmé verglichen mit der durch die heute üblichen Methoren der Wärme-

1) Die Konstruktion siehe bei Kowarschik, Dis Diathermie. Berlin 1913. Hier auch umfassende Literaturangabe. applikation erreichbaren. Es wurden also einmal mit etwa $3 \%$ iger warmer Kochsalzlösung angefeuchtete Elektroden, die aus einem mit Moos und Filz- oder Sackleinenschicht umgebenen feinverzweigten Metalldrahtnetz bestanden, seitlich angelegt und der Strom eingeschaltet; die genau dosierbare, an einem parallel zum Stromkreis eingeschalteten Hitzdrahtampèremeter dauernd kontrollierte Stärke betrug $1-11 \frac{1}{2} \mathrm{Am}$ père, nicht mehr, als wir sie auch therapeutisch anwandten. Es wurde dabei geachtet auf die Zeit, den Grad der Erwärmung und ganz besonders, ob überhaupt und in welchem Maße eine Wirkung in die Tiefe festzustellen war. Zum Vergleich wurden herangezogen heiße Breiumschläge von Leinsamenmehl, wie wir sie täglich auf der Station benutzen, dann nach Vorschrift erhitzte Thermophore von gleicher Größe, die bekanntlich einmal angenommene Wärme stundenlang halten; ferner ein. Stangerotherm in Form einer Halsmanschẹtte, die an den gewöhnlichen Wechselstrom angeschlossen wurde. Während des Versuches wurde dauernd die Temperatur an der Oberfläche des Modells festgestellt und auch die, welche sich tief im Innern entwickelte, also an Stellen, die mit den Thermophoren etc. garnicht in Berührung kamen.

Die Temperatur wurde gemessen mit Quecksilberthermometern. Dem Einwand, das Quecksilber werde infolge seines größeren Widerstandes durch den hochfrequente 0 Strom stärker erwärmt als die Umgebung und gebe also zu hohe Zahlen an, begegneten wir dadurch, daß wir sehr oft das Thermometer erst dann einschoben, wenn der Strom wieder ausgeschaltet war; diese und auch vergleichende Messungen mit einem Alkoholthermometer ergaben speziell bei der Diathermie keine oder nur so geringe Differenzen, da $\boldsymbol{B}$ man sie für unsere $Z$ wecke vernach lässigen kann.

Die Resultate waren nun folgende:

Wenn wir seitlich Breiumschläge auflegten, so stieg die Temperatur im Innern des Modells nach zwei Stunden auf $40^{\circ} \mathrm{C}$, nach drei auf etwa $50^{\circ} \mathrm{C}$, an der Oberfläche zwischen Breiumschlag und äußerer Gipshülle betrug sie $60-80^{\circ}$. Mit Thermophoren stieg sie in drei Stunden im Inneren auf $30^{\circ}$ bei einer Temperatur von $50^{\circ}$ an der Oberflä che; mit dem Stangerotherm betrug das Maximum im Inneren nach vier Stunden $50^{\circ}$ und an der Ober-

fläche etwa $100^{\circ}$. Mit- Kurve 1. Kleines Gipsmodell. tels Diathermie dagegen, wobei Elektroden von gleicher Größe wie die Thermophore angelegt wurden, stieg dieTemperatur schon in einer halben Stunde im Inneren auf über $50^{\circ}$, zwischen der Elektrode und Oberfläche nicht über $\mathbf{4 4}^{\circ}$ (s. Kurve 1).

Bei dem zweiten Gipsmodell, genau so konstruiert, nur war die Watteschicht doppelt so dick wie bei dem ersten, stieg die Temperatur im Inneren mittels Diathermie in zwei Stunden auf $\mathbf{4 3}^{\circ}$, betrug an der Oberfläche maximal $28^{\circ}$, mit Breiumschlägen und Stangerotherm in annähernd vier Stunden dagegen nur anf $30^{\circ}$ bei einer Oberflächentemperatur von $74^{0}$ und mit Thermophoren nur auf $24^{0}$ (s. Kurve 2).

Wurde die Watte zwischen beiden Gips. hüllen mit warmer Kochsalzlösung gut durchtränkt, so stieg die Temperatur bei der Thermopenetration im Inneren lang. samer, hieit aber auch doppelt so lange an.

Bei der Diathermie tritt also die Erwärmung im Inneren einmal in viel kürzerer Zeit, anderseits in höherem Grade ein als bei allen anderen Wärmeapplikationen, ohne daß die oberflächliche Temperatur unerträglich würde; sie dringt trotz des schlechten Wärmeleiters schneller und intensiver in die Tiefe, und zwar nur auf dem Elektrodenquerschnitt, wie Parallelmessungen in der Nähe ergaben.

Aehnliche Versuche wurden angestellt am lebenden Organismus, und zwar am Menschen; von Tierversuchen haben wir abgesehen, schon deshalb; weil ihre Resultate sich nicht ohne weiteres auf den Menschen übertragen lassen; anderseits sind beim letzteren der Wärmeapplikation auch gewisse Schranken 
gesetzt, weil man unerwünschte Nebenwirkungen wie Verbrennungen etc. vermeiden muß.

Einem jungen Manne wurde in die Hohlhand der fest zusammengeschlagenen Hände das Thermometer gelegt und auf die beiden Handrücken die Wärme appliziert. Nach mehrstïndigem Auflegen von Breiamschlägen, die häufig erneuert wurden, Thermophoren und Stangerotherm traten in der Hollhand nur ganz unbeträchtliche Temperatursteigerungen auf, als Maximum 0,20 gegen stieg die Temperatur unter gleichen Bedingungen in einer halben Stunde auf $39^{0}$ bei $1 \frac{1}{4}$ Ampère, auf $39,6^{0}$ bei $1 \frac{1}{2}$ Ampère, also um $2 \frac{1}{2^{0}}$,

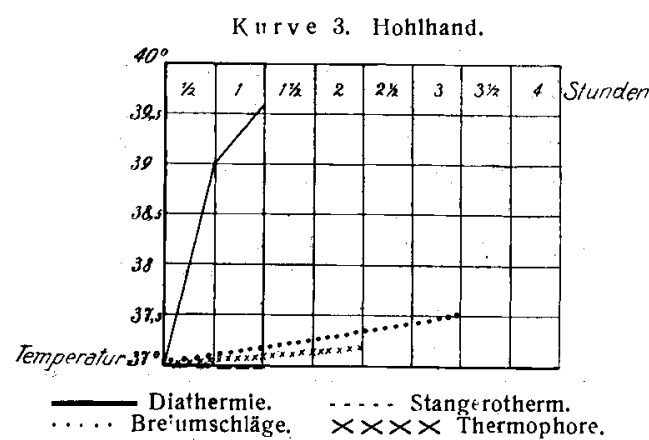
ohne daß der Mann unangenehme Nebenerscheinungen bot; er empfand nur ein angenehmes Wärmegefühl, das sich schließ lich in geringerem Grade über den ganzen Körper verbreitete, und nichts von einem elektrischen Strom (s. Kurve ¿).

Einem anderen ebenfalls fieberfreien Menschen wurden die Breiumschläge etc. auf die Glutäalgegend neben die Trochænteren appliziert und die Temperatur im Rectum kontrolliert. Nach einstündiger Diathermie stieg sie bei $11 / 4$ Ampère auf $38,5^{\circ}$, während die anderen Versuche auch bei mehrstündiger Anwendung nur ganz geringe Steigerung bewirkten, die stärkste mit dem Stangerotherm betrug $0,4^{0}$ (s. Kurve 4).

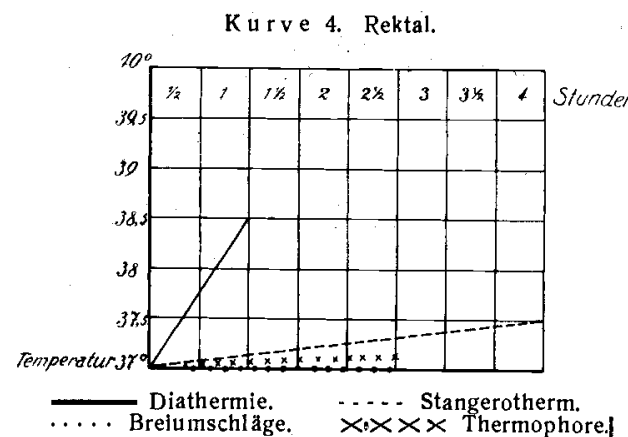

Genau so verfahren wurde bei einem fieberfreien Mann, der am

Oberschenkel eine große Fistel hatte in folge eines Senkungs. abszesses; das Thermo meter wurde $10 \mathrm{~cm}$ tie bis auf den Femur eingesteckt und die Breiumschläge etc. resp. Elektroden an der Außen- und Innenseite fixiert. Mit den gewöhnlichen Methoden trat nach mehrstündiger Anwendung als höchstes in der Fistel eine Temperatursteigerung voll $0,2^{0}$ auf, während sie mit dem Hochfrequenzstrom bei $11 / 4$ Ampère nach einer halben Stunde $1,5^{0}$ betrug, also $38,6^{0}$; auch in diesem Versuche gab der Patient nach etwa 20 Minuten spontan an, er fühle eine allgemeine, behagliche Wärme im ganzen Körper (s. Kurve 5).

Kurve 5. Oberschenkelfistel.

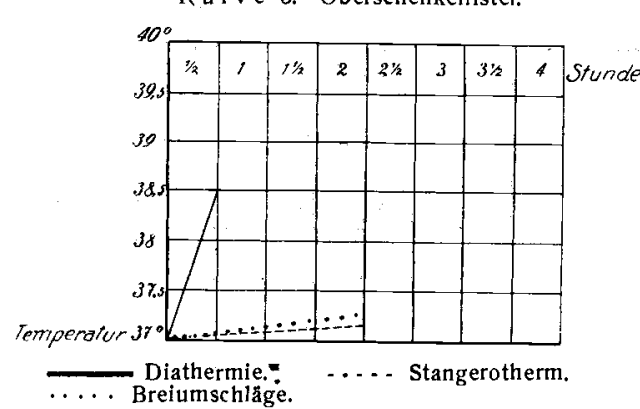

Andere Versuche mit Diathermie wurden gemacht bei zwei Männern mit Darm. fisteln nach Appendicitis-Operation resp. Resektion des Rectums, wobei das Thermometer tief in den Darm eingescho. ben wurde. Auch hier trat deutlich Temperatursteigerung auf, doch sind die Fälle weniger eklatant; es

waren dies die ersten Versuche zu einer Zeit, als wir uns in dje Technik des Verfahrens noch nicht genügend eingearbeitet hatten. Hierher gehört auch der einzige Fall, bei dem wir keine wesent liche Temperaturerhöhung erzielten; es handelte sich um ein schwertuberkulöses Kniegelenk mit hochgradigen, destruktiven Veränderungen und sehr stark sezernierenden Fisteln.

Diese Versuche ergaben also die stärkste Wärmeentwicklung in der Tiefe durch die Diathermie, sowohl am Modell wie beim Menschen.

Mit anderen Methoden der Wärmeapplikation (Stangerotherm) ließen sich zwar an Gipsmodell auch ähnliche Tenıperatursteigerungen im Inneren erreichen (bis 30 resp. $50^{\circ}$ ) jedoch mußte sie dann an der Oberfläche in einem Grade angewandt werden; die für die menschliche Haut unerträglich ist (bis 74 resp. $100^{\circ}$ ). Solange sie auf erträglicher Höhe ge- halten wurde trat eine wesentliche Erhöhung in der Tiefe nicht ein.

Eine deutliche, intensive Tiefenwirkung, bei der die Oberflächentemperatur noch erträglich war, lie $\beta$ sich nur mit der Diathermie erreichen. Auch die erforderliche Zeit war hier im Vergleich zu allen anderen Methoden wesentlich kürzer.

Die Versuche am Menschen ergaben ganz eindeutige Resultate. Bei der Diathermie wird zwar ein großer.Teil der zugeführten Wärme auf dem ganzen vom Strom durchflossenen Gebiet durch die Blut- und Lymphzirkulation dauernd fortgeführt - was ja auch schon die meist spontane Angabe der Betreffenden bestätigt, daß nämlich die subjektiv empfundene Wärme sich am Schluß der Sitzung über den ganzen Körper ausbreitet - aber trotzdem ist die lokale Erwärmung auf dem Elektrodenquerschnitt auch in der Tiefe ganz erheblich, während sie durch Breiumschläge, Thermophore und Stangerotherm nur gering ist oder ganz fehlt. Hinsichtlich der Intensität und auch der nötigen Zeit, vor allem aber in der Tiefenwirkung übertrifft also die Diathermie alle anderen Methoden bei weitem. Dabei ist sie für den Menschen an der Hautoberfläche im Bereich der Applikation sehr gut erträglich; gute Technik vorausgesetzt, fehlen unerwünschte Nebenwirkungen, speziell auch das Gefühl des elektrischen Stromes. Das Empfinden intensiver Wärme wird meist spontan angegeben, verbreitet sich schließlich in geringerem Grade auch über den ganzen Körper und hält sehr oft nach Ausschalten des Stromes und Abnahme der Elektroden an dem betreffenden Körperteil noch stundenlang an, ein Effekt, der bei jeder anderen Wärmeapplikation fehlt. Wir besitzen also in der Diathermie ein Verfahren, in verhältnismäßig kurzer Zeit tief im Körperinneren eine ganz erhebliche, genau kontrollierbare Wärme zu erzeugen ohne schädlichè Nebenwirkungen, eine Wärme, die auch an der Applikationsstelle gut ertragen wird, wie sie mit keinem anderen $\mathrm{zu}$ erreichen ist. Ob sie dabei auch noch andere Wirkungen entfaltet, konnten wir bei unserer Versuchsart nicht mit Sicherheit feststellen, ist aber doch sehr wahrscheinlich. Man kann wohl annehmen, da 3 mit der lokalen, auch in die Tiefe eindringenden, intensiven Erwärmung zugleich eine regere und bessere Blutzirkulation einhergeht, die ihrerseits wieder den Stoffwechsel anregt und günstig beeinflußt Wir haben auch in diesem Sinne einige Versuche angestellt, jedoch war die Wirkung dabei weniger deutlich.

Bei Männern mit gesundem Verdauungstraktus wurde nach einem Ewald schen Probefrühstiick die Magensaftsekretion geprüft. Im ersten Fall wurde viermal an jedem zweiten Tag ein Probefrühstiick gegeben und nach Ablauf der gleichen Zeit ausgehebert; beim zweiten und vierten Mal wurde der Magen 40 Minuten penetriert bei einer Stromstärke von $1 / 2$ resp. $3 / 4$ Ampère. Der Magen wurde möglichst vollständig entleert, dann die Gesamtazidität und freie Salzsäure bestimnt. Bei einem zweiten Patienten wurde genau so verfahren.

Eine deutliche Differenz in dem Resultat zugunsten der Diatherinie in Sinne einer stärkeren HCl-Absonderung haben wir nicht feststellen können. Aber damit ist ja nicht erwiesen, daß die Diatherrie ganz ohne Einfluß auf die Magensekreticn ist, dafür ist die Reihe der Momente, namentlich psychischer Art, welche sie beeinflussen, zu groß. Auch den Einfluß der Diathermie auf die Herztätigkeit gesunder Leute versuchten wir zu erfahren. ")

Zwei Männern mit gesundem Zirkulationsapparat wurde der Hochfrequenzstrom mittels entsprechend angelegten Elektroden so durch den Körper geleitet, daß das Herz in den Elektrodenquerschnitt zu liegen kam, und dieses Verfahren zur Kontrolle einige Male wiederholt. Weder der Blutdruck noch die Qualität und Frequenz des Pulses wurden durch halbstïndige Sitzungen bei $1 / 2-1$ Ampère Stromstärke irgendwie merklich beeinflußt; nur subjektiv wurde jedesmal ein angenehmes, intensives $\mathrm{Ge}$ fühl von Wärme in der Brusthöhle spontan angegeben.

Es kam uns bei unseren therapeutischen Erfahrungen nicht an auf eine möglichst große Anzahl als vielmehr auf die Art der Fälle, speziell den Krankheitsverlauf. Wir wählten solche aus, die-wir wegen chronischer Erkrankung lange Zeit auf der. Abteilung beobachtet und die allen angewandten sonst üblichen therapeutischen Maßnahmen gegenüber sich refraktär verhalten

1) Siehe den Vortrag v. Braunwarth, diese Wochenschrift 1913 , S. 337 bis 340 mit ausführlicher Diskussion 
hatten. Wir wollten ein Urteil darüber gewinnen, ob die Diathermie mehr leistete als andere Methoden, resp. ob sie dann noch einen günstigen Erfolg brachte, wenn alles andere versagt hatte.

Die Diathermiebehandlung wurde täglich einmal vorgenommen, zunächst im Verlauf einiger Minuten mit der Stromstärke langsam gestiegen und dann auf der Höhe 30, nur in wenigen Fällen 20 Minuten geblieben. Die Stromstärke schwankte in den einzelnen Krankheitsfällen zwischen 1 und $11 / 2$ Ampère, meist betrug sie $1 \frac{1}{4}$, je nachdem es der betreffende Körperteil vertrug; die mit warmer Kochsalzlösung angefeuchteten Elektroden wurden in der nötigen Größe auch hier so auf die Haut appliziert, daß der zu erwärmende Teil in den Elektrodenquerschnitt $\mathrm{zu}$ liegen $\mathrm{kam}$. Abgesehen von einigen geringfügigen Hautverbrennungen ersten Grades, die uns leider anfangs durch schadhafte Elektroden passierten, haben wir sonst jede Schädigung vermißt, sowohl auf der Haut als auch im Inneren; üble, unerwünschte Nebenwirkungen haben wir nie gesehen. Sie lassen sich auch vermeiden, wenn gewisse Vorsichtsma Bregeln beachtet werden. Nach unserer und auch anderer Meinung ist das Vertrautsein mit der Technik die Hauptsache; wer sie beherrscht und die Behandlung nicht Unerfahrenen überläßt, schützt auch seine Patienten vor Schädigungen. Ganz besondere Aufmerksamkeit erfordern die Elektroden; sie genügen leider noch nicht allen Anforderungen, der geringste Defekt des Drahtnetzes hat zur Folge, da $B$ die Wärmeentwicklung an umschriebener Stelle $\mathrm{zu}$ intensiv wird. Die Kranken machen zu gerne eine Abwehrbewegung; dadurch wird die Elektrode von der Unterlage (der Haut) entfernt, und so entstehen leicht umschriebene Verbrennungen der Haut. Die feuchten Elektroden müssen unter allen Umständen fest aufliegen und gut fixiert sein ${ }^{1}$.) Durch den Apparat selbst bedingte Störungen treten bei richtiger Handhabe höchst selten auf, es handelt sich dann meist nur um eine Unterbrechung des Stromkreises, die infolge der Konstruktion ohne schädliche Wirkung verläuft. Die Kranken unterzogen sich der Behandlung alle sehr gern, empfanden dabei ein intensives, gut zu ertragendes Gefühl von Wärme, auch im Inneren, das sich häufig gegen Schlu $B$ der einzelnen Sitzungen über den ganzen Körper verbreitete und namentlich an den behandelten Teilen oft stundenlang anhielt.

Der gute Erfolg mit der Diathermie bei akuten Gelenkentzündungen, besonders gonorrhoischer Aetiologie, ist ja bekannt. Wir haben nur chronische Arthritiden behandelt. Bei einer gonorrhoischen im Handgelenk war das Resultat nach 14 Tagen zufriedenstellend, bei drei anderen wurde nach 12-19 Sitzungen abgebrochen, weil jede Besserung ausblieb.

Zwei chronische Nephritiden - es waren beides sogenannte Mischformen - wurden lange behandelt; der eine Patient 18, der andere 30 Tage rechts und links je eine halbe Stunde. Eine kleine Elektrode wurde neben der Wirbelsäule in die Nierengegend, eine große vorn entsprechend auf das Abdomen gelegt. Irgendein Erfolg wurde nicht erzielt; jedenfalls „,eine an Heilung grenzende Besserung“, wie sie Nagelsch mid t (2) in zwei Fällen sah, war nicht zu konstatieren; weder schwanden die Oedeme, noch sank der Blutdruck, und auch im Urinbefund trat keine Aenderung ein, der Eiweißgehalt und der mikroskopische Befund war der gleiche wie vorher. Dagegen wurde eine Pyelitis nach sieben Sitzungen subjektiv frei von Beschwerden, und objektiv waren alle Symptome geschwunden.

Zwei ausgesprochen chronische Bronchitiden ohne Fieber, mit reichlichem Auswurf und ausgedehntem Lungenbefund, wurden ebenfalls lange behandelt, eine 34, die andere sogar 50 Tage. Vorn auf die Brust wurde eine mittelgroße, hinten eine große Elektrode appliziert. Weil subjektiv und objektiv jede Besserung ausblieb, wurde von dieser weiteren Behandlung abgesehen.

Günstiger waren die Erfolge bei chronischen Pneumonien; eine im linken Oberlappen zeigte nach 24 Sitzungen klinisch keine wesentliche Besserung, eine zweite im rechten Unterlappen dagegen löste sich in drei Wochen, eine dritte links schon in zwölf Tagen. Das günstige Resultat im klinischen Befund wurde jedesmal durch das Röntgenbild bestätigt.

Die besten Resultate mit der Thermopenetration sahen wir bei der Pleuritis exsudativa; sie wurde angewandt in vier Fällen, bei denen das Exsudat auch im Rötngenbild lange Zeit stationär geblieben war, trotz der verschiedensten therapeutischen Maßnahmen. Mit dem Be-

1) Was sich durch entsprechend angelegte Flanellbinden einfach und gut erreichen läßt. ginn der Diathermie wurde auch hier jede andere Behandlung ausgesetzt; der Erguß war zufällig jedesmal links; hinten wurde eine mittelgroße, vorne eine kleine Elektrode aufgelegt. Bei zwei Patienten resorbierte sich das Exsudat in zwölf, einmal sogar schon in sieben Tagen. Bei dem vierten Patienten blieb das Exsudat trotz Diathermiebehandlung unverändert, aber in der dritten Woche schwand das Fieber, das beim Beginn der Thermopenetration schon sieben Wochen lang unverändert zwischen 38 und $39^{\circ}$ geschwankt hatte; subjektiv fühlte sich der Patient bei der Diathermie wesentlich besser; nach 24 Sitzungen wurde, weil klinisch und röntgenologisch sich keine Veränderung zeigte, diese $\mathrm{Be}$ handlung ausgesetzt und das seröse Exsudat abgelassen. Nun traten wieder bald Temperatursteigerungen auf.

Die therapeutischen Erfolge bei ausgesprochen chronischen Erkrankungen sind also teils recht gut, teils blieben sie überhaupt ganz aus. Den Grund für die schlechten Resultate anzugeben, ist schwierig. Vielleicht liegt er in dem Alter der Fälle selbst; auch wäre es denkbar, daß der hochfrequente Strom in einzelnen Fällen nicht den erforderlichen Weg durch das zu erwärmende Organ nimmt, zumal, wenn er auf lufthaltige Organe stößt, wie z. B. Lunge und Darm. Infolge ihrer schlechten Leitfähigkeit für den Diathermiestrom läßt er sie unberührt, und eine intensive Wärmeentwicklung an der gewünschten Stelle bleibt aus. Von diesem Gesichtspunkt aus wäre es dann auch verständlich, daß andere Erkrankungen des Brustraumes, wie die chronische Pneumonie und das Pleuraexsudat, besser reagierten auf die Thermopenetration, weil sie dem Strom einen größeren Widerstand entgegensetzen und so intensiver erwärmt werden. Die Behandlung mit Hochfrequenzströmen ist eben noch im Stadium der Entwicklung, manche Frage ist noch ungelöst. Erst die weitere Anwendung und Erfahrung wird lehren, welche Erkrankungen innerer Organe sich am besten eignen für die Diathermiebehandling. Aber soviel steht heute fest: wir besitzen in ihr eine Methode der unschädlichen Wärmeapplikation, die alle bisherigen an Intensität und Tiefenwirkung bei weitem übertrifft und auch solche chronische Erkrankungen noch günstig beeinfluBt und sogar zur klinischen Heilung bringt, bei denen alle anderen Maßnahmen nicht zum Ziele führten.

Liter a tur: 1. Wiener klinische Wochenschrift 1908, Nr. 15, und 1909, Nr. 44. 2. Diese Wochenschrift 1911, Nr. 1. - 3. Zeitschrift für arztliche Fortbildung 1910, und 2. Diese Wochenschrift 1911, Nr. 1. - 3. Zeitschrift für arztliche Fortbildung 1910, und Therapie det Gegenwart 1911. - 4. Therapeutische Monatshefte 1911, und Kongreß
Wiesbaden 1911. -5 . Berliner klinische Wochenschrift 1909, No.39. - 6. Medizinische Wiesbaden 1911. -5 . Berliner klinische Wochensch
Klinik 1910, Nr. 35 u. $36 .-7$. Ebenda 1913, Nr. 19. 